\title{
ON MAXIMAL NILPOTENT SUBRINGS OF RIGHT NOETHERIAN RINGS
}

\author{
by GERHARD MICHLER $\dagger$ \\ (Received 30 July, 1966)
}

1. Introduction. Applying Hopkins's Theorem asserting that each unitary right Artinian ring is right Noetherian, G. Köthe and K. Shoda proved the following theorem (cf. Köthe [7], p. 360, Theorem 1 and p. 363, Theorem 5): If $R$ is a unitary right Artinian ring, then the following statements hold:

(i) Each nilpotent subring of $R$ is contained in a maximal nilpotent subring of $R$.

(ii) The intersection of all maximal nilpotent subrings of $R$ is the maximal nilpotent twosided ideal of $R$.

(iii) All maximal nilpotent subrings of $R$ are conjugate.

Our problem is to decide which of these statements remain valid in right Noetherian rings. It is an immediate consequence of the Theorem of Levitzki (cf. Jacobson [6, p. 199, Theorem 1]), and Theorem 1 of Herstein and Small [5, p. 775], that each nilpotent subring of a right Noetherian ring $R$ is contained in a maximal nilpotent subring of $R$ (Proposition 1).

In [1] D. W. Barnes proved statement (ii) for all rings with minimum condition for right ideals. Now, if $R$ is any right Artinian ring, then the sum $B(R)$ of all nilpotent ideals of $R$ is nilpotent, and $R / B(R)$ is a unitary right Artinian and right Noetherian ring. Hence Barnes's Theorem is a consequence of the following theorem:

If $R$ is a ring such that the sum $B(R)$ of all nilpotent ideals of $R$ is nilpotent, and that $R / B(R)$ is right Noetherian, then statement (ii) holds in $R$.

This theorem is an easy consequence of our Theorem 1. It is perhaps remarkable that we do not make full use of the maximum condition for right ideals of $R / B(R)$. In particular, it follows that the intersection of all maximal nilpotent subrings of a right Noetherian ring $R$ is the maximal nilpotent ideal $B(R)$ of $R$ (Corollary 4 ).

In rings without an identity element the customary concept of conjugacy is not applicable. Consequently we term the subrings $X$ and $Y$ of $R$ quasi-conjugate if there exists a pair of elements $u, v$ in $R$ satisfying $u+v=u v=v u$ such that $Y$ is the totality of elements $x-u x-x v+u x v$ with $x$ in $X$. Then our Theorem 2 asserts: If $R$ is a right Artinian ring without additive subgroups of type $p^{\infty}$, then its maximal nilpotent subrings are quasiconjugate.

† Research supported in part by the European Research Office, US Department of the Army, Frankfurt am Main, Contract No. DA-91-591-EUC-3339-452-3298. 
2. Notations and definitions. Throughout this paper, every ring $R$ is associative. The existence of an identity in $R$ is not assumed.

$R^{+}=$additive group of the ring $R$.

$A \oplus B=$ ring-theoretical direct sum of the ideals $A, B$ of $R$.

$A+B=$ direct sum of the right ideals $A, B$ of $R$.

$\sum_{\mu \in I}^{\mathrm{d}} R_{\mu}=$ discrete direct sum of the rings $R_{\mu}$.

$\sum_{\mu \in I}^{c} R_{\mu}=$ complete direct sum [or Cartesian sum] of the rings $R_{\mu}$.

If $M$ is a subset of $R$, then we denote by $(M)_{R}$ the right ideal of $R$ generated by $M$.

Let $X$ be a subset of the ring $R$. Then

$$
\begin{gathered}
X_{l}(R)=\{x \in R \mid x X=0\}, \quad X_{r}(R)=\{x \in R \mid X x=0\} . \\
Z^{r}(R)=\left\{x \in R \mid x_{r}(R) \text { is an essential right ideal of } R\right\}=\text { right singular ideal of } R .
\end{gathered}
$$

The subrings $X$ and $Y$ of the ring $R$ are quasi-conjugate if there is a quasi-regular element $u \in R$ such that

$$
Y=\{x-u x-x v+u x v \mid x \in X, \text { where } v \text { is the quasi-inverse of } u \text { in } R .\}
$$

Right Goldie ring $=$ ring with ascending chain condition on right annihilators and on direct sums of right ideals.

The right ideal $U \neq 0$ of the ring $R$ is called uniform if $X \cap Y \neq 0$ for all right ideals $X \neq 0 \neq Y$ of $R$ with $X, Y \leqq U$.

If $R$ is a semi-prime right Goldie ring, then, by Goldie [4, p. 202, Theorem, 1.1], there is a positive integer $n$ such that

(i) every direct sum of uniform right ideals of $M$ contains at most $n$ terms,

(ii) a right ideal $X$ of $R$ is essential if and only if $X$ contains a sum of $n$ uniform right ideals. The integer $n$ is called the dimension of $R$ and is denoted by $\operatorname{dim} R$.

The right ideals $U, V$ of $R$ are subisomorphic if there is a right $R$-module monomorphism $\theta$ of $U$ into $V$, and a right $R$-module monomorphism $\mu$ of $V$ into $U$.

3. Maximal nilpotent subrings of rings with certain chain conditions. Let $\mathfrak{n}$ be a nonempty set of subrings $S$ of the ring $R$ such that the union of every tower $t$ of $n$ belongs to $n$. Then by Zorn's Lemma we obtain that each n-subring of the ring $R$ is contained in a maximal $\mathfrak{n}$-subring of $R$. If $\mathfrak{n}$ is the set of all locally nilpotent (resp. nil) subrings of $R, \mathfrak{n}$ satisfies our transfinite induction hypothesis. Thus we have

LEMMA 1. (a) Each locally nilpotent subring of an arbitrary ring $R$ is contained in a maximal locally nilpotent subring of $R$.

(b) Each nil subring of an arbitrary ring $R$ is contained in a maximal nil subring.

By Herstein and Small [5, p. 775, Theorem 1], every nil subring of a ring $R$ satisfying the ascending chain conditions on right and left annihilators is nilpotent. This implies 
COROLLARY 1. If $R$ is a ring with maximum conditions on right and left annihilators, then each nilpotent subring is contained in a maximal nilpotent subring.

Now let $R$ be a right Noetherian ring. Then the sum $B(R)$ of all nilpotent ideals of $R$ is nilpotent by Levitzki's Theorem (cf. Jacobson [6, p. 199, Theorem 1]). Since $R / B(R)$ is a semi-prime right Noetherian ring, it satisfies the maximum conditions on right and left annihilators by Procesi and Small [12, p. 81, Lemma 2]. Hence from Corollary 1 and Lemma 1 we deduce

Proposition 1. Each nilpotent subring of a right Noetherian ring $R$ is contained in $a$ maximal nilpotent subring of $R$.

In the following it will be proved that the intersection of all maximal nilpotent subrings of a right Noetherian ring $R$ is just the sum $B(R)$ of all nilpotent ideals of $R$.

Lemma 2. Let $M \neq 0$ be a nilpotent subring of the prime right Goldie ring $R$. Let $r$ be the exponent of $M$ and $n=\operatorname{dim} R$. Then

(a) $r \leqq n$,

(b) $\operatorname{dim}(M)_{R} \leqq n-1$,

(c) there exists a set of $r$ idempotents $e_{0}=0, e_{k} \neq 0(k=1,2, \ldots, r-1)$ of the ring $Q$ of right quotients of $R$ satisfying $e_{k} Q=M^{r-k} Q$ such that

$$
M \leqq R \cap \sum_{k=1}^{r-1}\left(e_{k}-e_{k-1}\right) Q\left(1-e_{k}\right)
$$

Proof. $(M)_{R}$ is a right ideal of $R$; so $\operatorname{dim}(M)_{R} \leqq \operatorname{dim} R=n$. Assume that $\operatorname{dim}(M)_{R}=n$; then $M Q=Q$ by Goldie [4, p. 212, Lemma 4.3]. Hence $0=M^{r} Q=Q \neq 0$. This contradiction proves $(b)$.

Since $M$ is a nilpotent subring of the unitary Artinian ring $Q$, which is a ring of $n \times n$ matrices over a division ring, the exponent $r$ of $M$ satisfies $r \leqq n$ by Levitzki [8, p. 625, Zusatz], and ( $a$ ) has been proved.

For each integer $1 \leqq m \leqq r-1$ we have $M^{m} Q>M^{m+1} Q$; for equality implies that $M=0$, because $Q$ has an identity element. If $d(r-i)=\operatorname{dim}\left(M^{r-i}\right)_{R}$ for $i=1,2, \ldots, r-1$, then there are primitive orthogonal idempotents $e_{i, j_{i}} \neq 0\left(i=1,2, \ldots, r-1, j_{i}=1,2, \ldots\right.$, $d(r-i))$ of $Q$ such that

$$
M^{r-i} Q=e_{1,1} Q+\ldots+e_{1, d(r-1)} Q+\ldots+e_{i, 1} Q+\ldots+e_{i, d(r-i)} Q .
$$

If $e_{k}=e_{1,1}+\ldots+e_{1, d(r-1)}+e_{2,1}+\ldots+e_{k, 1}+\ldots+e_{k, d(r-k)}$ for $k=1,2, \ldots, r-1$, then we obtain $e_{k} e_{k+1}=e_{k}=e_{k+1} e_{k}$, because the $e_{i, j_{1}}$ are orthogonal idempotents. Furthermore we have $M^{r-k} Q=e_{k} Q$. From $e_{k+1} Q=M^{r-k-1} Q>M^{r-k} Q=e_{k} Q$ for all $k=1,2, \ldots, r-1$ we deduce for each $a \in M$ that

$$
a\left(e_{k+1} Q\right)=a M^{r-k-1} Q \leqq M^{r-k} Q=e_{k} Q .
$$


Hence $a e_{k+1}=e_{k} q \in e_{k} Q$ for some $q \in Q$. Since

$$
\left(e_{k+1}-e_{k}\right) a e_{k+1}=\left(e_{k+1}-e_{k}\right) e_{k} q=e_{k+1} e_{k} q-e_{k} q=e_{k} q-e_{k} q=0,
$$

it follows that $\left(e_{k+1}-e_{k}\right) a \in\left(e_{k+1}\right)_{1}(Q)=Q\left(1-e_{k+1}\right)$. Therefore

$$
\left(e_{k+1}-e_{k}\right) a \in\left(e_{k+1}-e_{k}\right) Q\left(1-e_{k+1}\right)
$$

is a consequence of $e_{k+1} e_{k}=e_{k}=e_{k} e_{k+1}$. Now $M \leqq M Q=e_{r-1} Q$ implies that $a=e_{r-1} a$. Hence

$$
a=\sum_{k=1}^{r-1}\left(e_{k}-e_{k-1}\right) a \in \sum_{k=1}^{r-1}\left(e_{k}-e_{k-1}\right) Q\left(1-e_{k}\right)
$$

where $e_{0}=0$. This completes the proof.

The nilpotent ring $M$ has exponent $h$, if $h$ is the least positive integer $r$ with $M^{r}=0$.

LemMA 3. Let $R$ be a prime right Goldie ring, and $Q$ its ring of right quotients. Then the following statements hold:

(a) There are $n=\operatorname{dim} R$ primitive orthogonal idempotents $g_{i} \neq 0$ of $Q$ such that $g_{k} Q \cap R \neq 0 \neq R \cap Q g_{k}$ for all $k=1,2, \ldots, n$.

(b) If the $f_{i} \neq 0(i=1,2, \ldots, n)$ are $n$ primitive orthogonal idempotents of $Q$ such that $f_{i} Q \cap R \neq 0 \neq R \cap Q f_{i}$ for all $i$, then

(i) $T=R \cap\left[\sum_{j=1}^{n-1} f_{j} Q\left(\sum_{h=j+1}^{n} f_{h}\right)\right]$ and $U=R \cap\left[\sum_{j=1}^{n-1}\left(\sum_{h=j+1}^{n} f_{h}\right) Q f_{j}\right]$

are maximal nilpotent subrings of $R$ with exponent $n$ satisfying $U \cap T=0$, and

(ii) if $N$ is a nilpotent subring of $R$ containing an element $t$ of the form

$$
t=\sum_{i=1}^{n-1} f_{i} q_{i} f_{i+1}
$$

where $0 \neq f_{i} q_{i} f_{i+1} \in R$ and $q_{i} \in Q$ for all $i=1,2, \ldots, n-1$, then $N \leqq T$, and $N$ has exponent $n$.

(c) For each nilpotent subring $M$ of $R$ there exists a regular element $c \in R$ such that $c^{-1} M c \cap R$ is contained in a maximal nilpotent subring $S$ of $R$ with exponent $n=\operatorname{dim} R$. $\left[c^{-1}\right.$ denotes the inverse of $c$ in $\left.Q.\right]$

Proof. By Goldie's Theorem for prime rings, $Q$ is a ring of $n \times n$ matrices over a division ring $D$. Hence there are $n$ orthogonal primitive idempotents $e_{i} \in Q$. Since $R$ is a classical right order of $Q$, each $e_{i}$ has the form $e_{i}=a_{i} c_{i}^{-1}$, where $a_{i}, c_{i} \in R$, and $c_{i}$ is regular. By Jacobson [6, p. 263, Lemma 1], there exist regular elements $b_{1}, b_{2}, \ldots, b_{n}, c \in R$ such that $c_{i}^{-1}=b_{i} c^{-1}$ for $i=1,2, \ldots, n$. If $g_{i}=c^{-1} e_{i} c$ for all $i$, then the $g_{i}$ form a maximal set of orthogonal primitive idempotents of $Q$. Clearly $R \cap g_{i} Q \neq 0$ for all $i$. This together with $0 \neq a_{i} b_{i}=c g_{i}$ proves $(a)$. 
Now let $f_{i} \neq 0(i=1,2, \ldots, n)$ be $n$ orthogonal primitive idempotents of $Q$ such that $f_{i} Q \cap R \neq 0 \neq R \cap Q f_{i}$ for all $i$. Since $R$ is a prime ring we have

$$
0 \neq\left(f_{i} Q \cap R\right)\left(Q f_{h} \cap R\right) \leqq f_{i} Q f_{h} \cap R \quad \text { for } i, h=1,2, \ldots, n .
$$

Let $x_{i}(i=1,2, \ldots, n-1)$ be $n-1$ elements of $Q$ such that $0 \neq f_{i} x_{i} f_{i+1} \in R$. Let $N$ be a nilpotent subring of $R$ containing the element

$$
x=\sum_{i=1}^{n-1} f_{i} x_{i} f_{i+1} .
$$

Clearly $x \neq 0$, because the $f_{i}$ are orthogonal primitive idempotents of $Q$. Now $x$ satisfies $x^{n}=0$. But $x^{n-1} \neq 0$, because

$$
0=x^{n-1}=\left(f_{1} x_{1} f_{2}\right)\left(f_{2} x_{2} f_{3}\right) \ldots\left(f_{n-2} x_{n-2} f_{n-1}\right)\left(f_{n-1} x_{n-1} f_{n}\right)
$$

would imply that

$$
\begin{aligned}
0=x^{n-1} Q & =\left(f_{1} x_{1} f_{2}\right)\left(f_{2} x_{2} f_{3}\right) \ldots\left(f_{n-2} x_{n-2} f_{n-1}\right)\left(f_{n-1} x_{n-1} f_{n}\right) Q \\
& =\left(f_{1} x_{1} f_{2}\right)\left(f_{2} x_{2} f_{3}\right) \ldots\left(f_{n-2} x_{n-2} f_{n-1}\right) Q=\ldots \\
& =\left(f_{1} x_{1} f_{2}\right) Q=f_{1} Q \neq 0,
\end{aligned}
$$

since $Q$ is an associative ring and the right ideals $f_{k} Q(k=1,2, \ldots, n)$ are minimal right ideals of $Q$. From $x^{n-1} \neq 0$ we obtain by Lemma 2 that $n=\operatorname{dim} R$ is the exponent of $N$. Therefore

$$
0<N^{n-1} Q<N^{n-2} Q<\ldots<N^{2} Q<N Q<Q
$$

is a (right) composition series of the unitary simple Artinian ring $Q$. Hence from

$$
0 \neq x^{n-s}=\sum_{j=1}^{s}\left[\left(f_{j} x_{j} f_{j+1}\right)\left(f_{j+1} x_{j+1} f_{j+2}\right) \ldots\left(f_{j+n-s-1} x_{j+n-s-1} f_{j+n-s}\right)\right] \in N^{n-s}
$$

for $s=1,2, \ldots, n-1$ we obtain $N^{n-s} Q=\left(f_{1}+f_{2}+\ldots+f_{s}\right) Q$ for all $s$. Therefore by application of Lemma 2 we have

$$
N \leqq R \cap\left[\sum_{j=1}^{n-1} f_{j} Q\left(1-\sum_{h=1}^{j} f_{h}\right)\right]=R \cap\left[\sum_{j=1}^{n-1} f_{j} Q\left(\sum_{h=j+1}^{n} f_{h}\right)\right]=T .
$$

This completes the proof of Lemma 3(b) (ii).

Now we have to show that $T$ is a maximal nilpotent subring of $R$. Since the $f_{i}$ are orthogonal idempotents, $T$ is a nilpotent subring of $R$. By Lemma 2 of Procesi and Small $[12$, p. 81] and Corollary 1 we know that $T$ is contained in a maximal nilpotent subring $S$ of $R$. From (3.1) we deduce the existence of $n-1$ elements $q_{i} \in Q$ such that $0 \neq f_{i} q_{i} f_{i+1} \in R$ for $i=1,2, \ldots, n-1$. Clearly $f_{i} q_{i} f_{i+1} \in T$ for all $i$. Thus

$$
t=\sum_{i=1}^{n-1} f_{i} q_{i} f_{i+1} \in T \leqq S .
$$

Hence, by Lemma $3(b)$ (ii), $S \leqq T$, and $T$ is a maximal nilpotent subring of $R$. 


$$
U=R \cap\left[\sum_{j=1}^{n-1}\left(\sum_{h=j+1}^{n} f_{h}\right) Q f_{j}\right] \text {, }
$$

then $U$ is a nilpotent subring of $R$, and, by the right-left symmetry of the given proof for $T$ being a maximal nilpotent subring of $R$, it can be shown that $U$ is a maximal nilpotent subring of $R$. Since the elements $f_{k}(k=1,2, \ldots, n)$ are orthogonal idempotents, it is easy to see that $U \cap T=0$. Thus Lemma 3(b) (i) holds.

Now let $M$ be a nilpotent subring of $R$ with exponent $r$. By Lemma 2 there exists a set of $r-1$ idempotents $e_{k} \neq 0(k=1,2, \ldots, r-1)$ of the ring $Q$ such that

$$
M \leqq R \cap\left(\sum_{k=1}^{r-1}\left(e_{k}-e_{k-1}\right) Q\left(1-e_{k}\right)\right),
$$

where $e_{0}=0$. By the proof of Lemma 2, each $e_{k}$ has the form

$$
e_{k}=e_{1,1}+\ldots+e_{1, d(r-1)}+e_{2,1}+\ldots+e_{k, d(r-k)},
$$

where $d(r-i)=\operatorname{dim}\left(M^{r-i}\right)_{R}$, and where the $e_{i, j_{i}} \neq 0\left[i=1,2, \ldots, r-1 ; j_{i}=1,2, \ldots, d(r-i)\right]$ are orthogonal primitive idempotents of $Q$. Clearly this set of orthogonal primitive idempotents of $Q$ can be extended to a maximal set of $n$ orthogonal primitive idempotents $f_{j} \neq 0(j=1,2, \ldots, n)$ of $Q$ such that

$$
e_{i, j_{i}}=f_{j_{i}}+\sum_{s=1}^{i} d(r-s-1)
$$

for $i=1,2, \ldots, r-1$. Hence

$$
M \leqq R \cap\left[\sum_{j=1}^{n-1} f_{j} Q\left(\sum_{h=j+1}^{n} f_{h}\right)\right]
$$

Each $f_{j}=x_{j} c_{j}^{-1}$, where $x_{j}, c_{j} \in R$, and $c_{j}$ is regular. By Jacobson [6, p. 263, Lemma 1] there are regular elements $b_{1}, b_{2}, \ldots, b_{n}, c \in R$ with $c_{j}^{-1}=b_{j} c^{-1}$. Let $g_{j}=c^{-1} f_{j} c$ for $j=1,2, \ldots, n$. By the proof of Lemma $3(a)$ the primitive orthogonal idempotents $g_{j}$ satisfy $g_{j} Q \cap R \neq 0$ $\neq Q g_{j} \cap R$ for all $j$. Hence

$$
c^{-1} M c \cap R \leqq R \cap\left[\sum_{j=1}^{n-1} g_{j} Q\left(\sum_{h=j+1}^{n} g_{h}\right)\right]=T .
$$

By Lemma $3(b)(\mathrm{i}), T$ is a maximal nilpotent subring of $R$ with exponent $n$.

This completes the proof of Lemma 3.

Remark 1. Let $R$ be a prime right Goldie ring, and $M$ be a maximal nilpotent subring of $R$. If $c \in R$ is a regular element of $R$, then in general the nilpotent subring $c^{-1} M c \cap R$ of $R$ is not a maximal nilpotent subring of $R$, as can be seen by the following 
Example. Let $K$ be a unitary principal right ideal domain which is not a left Ore domain (e.g. Goldie [4, p. 219]). Hence there are $x, y \in K$ such that $K x \cap K y=0$. Let $R$ be the ring of all $2 \times 2$ matrices $\left(a_{i j}\right)(i, j=1,2)$ with $a_{1 j} \in K x$ and $a_{i 2} \in K y$. If $\hat{R}$ is the division ring of right quotients of $K$, then $Q=\widehat{R}_{2}$ is the classical ring of right quotients of $R$, by Faith and Utumi [2, p. 59]. Hence $R$ is a prime right Goldie ring (cf. Jacobson [6, p. 268], Goldie's Theorem for prime rings). Let $M$ be the ring of all matrices $\left(a_{i j}\right)$ of $R$ with $a_{11}=a_{12}=a_{22}=0$ and $a_{21} \in K x$. We shall prove that $M$ is a maximal nilpotent subring of $R$. If this were not true, then there would be a $v \in R, v \notin M$ such that $\{M, v\}$ is a nilpotent subring of $R$. By Lemma 2 we have $\{M, v\}^{2}=0$. Let

$$
v=\left(\begin{array}{ll}
a & b \\
c & d
\end{array}\right) \text { and } g=\left(\begin{array}{ll}
0 & 0 \\
c & 0
\end{array}\right)
$$

where $a, c \in K x$ and $b, d \in K y$. Then $v-g \in\{M, v\}$. Thus $(v-g)^{2}=0$. Hence $a=0=d$. From $v^{2}=0$ we deduce that $c=0$ or $b=0$. If we had $b \neq 0$, then we would obtain $v+z \in\{M, v\}$ for

$$
z=\left(\begin{array}{ll}
0 & 0 \\
x & 0
\end{array}\right)
$$

Therefore $(v+z)^{2}=0$ which implies that $b x=0$. Hence $b=0$, and we get $v=g \in M$, a contradiction. Therefore $M$ is a maximal nilpotent subring of $R$. If $N$ is the ring of all matrices $\left(a_{i j}\right) \in R$ with $a_{11}=a_{21}=a_{22}=0$ and $a_{12} \in K y$, then by a similar argument $N$ also is a maximal nilpotent subring of $R$. Clearly

$$
c=\left(\begin{array}{ll}
0 & y \\
x & 0
\end{array}\right)
$$

is a regular element of $R$, and $c^{-1} M c \cap R \leqq N$. If $c^{-1} M c \cap R$ were a maximal nilpotent subring of $R$, then we would have $c^{-1} M c \cap R=N$. Hence there would be an element $w \in K x$ with $y^{-1} w y=y$, and thus we would have $0 \neq y=w=k x \in K x \cap K y=0$. This contradiction shows that $c^{-1} M c \cap R<N$.

It is well known (cf. Goldie [4], p. 206, Theorem 3.2) that each semi-prime right Goldie ring has the following properties:

(a) $Z_{r}(R)=0$.

(b) Each set of independent uniform right ideals of $R$ which are subisomorphic in pairs has a finite number of elements.

(c) Each right ideal $X \neq 0$ of $R$ contains a uniform right ideal $U \neq 0$ of $R$.

We therefore call a ring $R$ with the properties $(a),(b)$ and $(c)$ a generalized right Goldie ring.

Lemma 4. Let $\mathrm{n}$ be a set of nil subrings of the semi-prime generalized right Goldie ring $R$ such that the union of each tower of n-rings is an n-ring. If each locally nilpotent subring $X$ of $R$ belongs to $\mathrm{n}$, then there exist two maximal $\mathrm{n}$-subrings $H$ and $G$ of $R$ with $H \cap G=0$. 
Proof. Since $R$ is a semi-prime ring with the properties $(a),(b)$ and $(c)$, by Lemma 4.2 and Lemma 4.5 of [10] $R$ is an irredundant subdirect sum of the prime right Goldie rings $R_{\mu}=R / P_{\mu}\left(\mu \in I, I\right.$ a well ordered index set), where the ideals $P_{\mu}$ are the maximal two-sided annihilator ideals of $R$. If $A_{\mu}=\left(P_{\mu}\right)_{r}(R)$, then $A_{\mu}$ is a minimal two-sided annihilator ideal of $R$ by L. Levy [9, p. 65, Lemma 3.1]. Since $R$ is semi-prime, $A_{\mu} \cap P_{\mu}=0$ for all $\mu \in I$. Hence $A_{\mu}$ is isomorphic to a two-sided ideal $\bar{A}_{\mu}$ of $R_{\mu}$. If we identify $A_{\mu}$ with $\bar{A}_{\mu}$, then $A_{\mu}$ is an essential right ideal of the prime right Goldie ring $R_{\mu}$. Hence $A_{\mu}$ is a prime ring with $Z^{r}\left(A_{\mu}\right)=0$, and $\operatorname{dim}_{R}\left(A_{\mu}\right)=\operatorname{dim}_{A_{\mu}}\left(A_{\mu}\right)=\operatorname{dim}_{R_{\mu}}\left(A_{\mu}\right)=\operatorname{dim} R_{\mu}$. Thus $A_{\mu}$ has a ring of right quotients $Q\left(A_{\mu}\right)$ which coincides with the ring of right quotients $Q_{\mu}$ of $R_{\mu}$, because $A_{\mu}$ is a two-sided ideal of $R$. Application of Lemma $3[(a)$ and $(b)(i)]$ shows the existence of a maximal set of primitive orthogonal idempotents $g_{i}^{(\mu)} \in Q_{\mu}\left(i=1,2, \ldots, n_{\mu} ; \mu \in I\right)$ such that

and

$$
T_{\mu}=A_{\mu} \cap\left[\sum_{j=1}^{n_{\mu}-1} g_{j}^{(\mu)} Q_{\mu}\left(\sum_{h=j+1}^{n_{\mu}} g_{h}^{(\mu)}\right)\right],
$$

$$
S_{\mu}=A_{\mu} \cap\left[\sum_{j=1}^{n_{\mu}-1}\left(\sum_{h=j+1}^{n_{\mu}} g_{h}^{(\mu)}\right) Q_{\mu} g_{j}^{(\mu)}\right]
$$

are maximal nilpotent subrings of $A_{\mu}$ with exponent $n_{\mu}$. Furthermore $T_{\mu} \cap S_{\mu}=0$.

Let $T=\sum_{\mu \in I}^{d} T_{\mu}$. Then $T$ is a nil subring of $R$ contained in $\sum_{\mu \in I}^{d} A_{\mu} \leqq R$. If $t_{i} \in T(i=1,2, \ldots$, $m$ ), where $m$ is any positive integer, then there is a finite number $z$ (depending on the $t_{i} \in T$ ) of minimal two-sided annihilators $A_{k}(k=1,2, \ldots, z)$ such that the subring $\left\{t_{1}, t_{2}, \ldots, t_{m}\right\}$ of $R$ generated by the $t_{i}$ is contained in $V=A_{1} \oplus A_{2} \oplus \ldots \oplus A_{z}$. Since $V$ is a complete direct sum of a finite number of prime right Goldie rings, $V$ is a semi-prime right Goldie ring. Hence the nil subring $\left\{t_{1}, t_{2}, \ldots, t_{m}\right\}$ of $V$ is nilpotent by application of Procesi and Small $[12$, p. 81, Lemma 2] and Herstein and Small [5, p. 775, Theorem 1]. Thus $T$ is a locally nilpotent subring of $R$. Hence $T$ is an $\mathfrak{n}$-subring of $R$. Since the union of each tower of n-rings is an n-ring, $T$ is contained in a maximal $n$-subring $G$ of $R$. If $S=\sum_{\mu \in I}^{d} S_{\mu}$, by the same argument we get that $S$ is contained in a maximal $\mathfrak{n}$-subring $H$ of $R$. From $G \geqq T$ and $H \geqq S$ we obtain $G_{\mu} \geqq T_{\mu}$ and $H_{\mu} \geqq S_{\mu}$ for all $\mu \in I$. For each $\mu \in I$ we define

and

$$
F_{\mu}=R_{\mu} \cap\left[\sum_{j=1}^{n_{\mu}-1} g_{j}^{(\mu)} Q_{\mu}\left(\sum_{h=j+1}^{n_{\mu}} g_{h}^{(\mu)}\right)\right],
$$

$$
E_{\mu}=R_{\mu} \cap\left[\sum_{j=1}^{n_{\mu}-1}\left(\sum_{h=j+1}^{n_{\mu}} g_{h}^{(\mu)}\right) Q_{\mu} g_{j}^{(\mu)}\right] .
$$

Clearly $F_{\mu} \cap E_{\mu}=0$ for all $\mu \in I$.

We now want to show that $G_{\mu} \leqq F_{\mu}$ and $H_{\mu} \leqq E_{\mu}$ for all $\mu \in I$, By Lemma 3(a) applied to the prime right Goldie ring $A_{\mu}$ we have $g_{k}^{(\mu)} Q_{\mu} \cap A_{\mu} \neq 0 \neq Q_{\mu} g_{k}^{(\mu)} \cap A_{\mu}$ for all $k=1,2, \ldots$, $n_{\mu}$ and all $\mu \in I$. Since each $A_{\mu}$ is a prime ring we get

$$
0 \neq g_{j}^{(\mu)} Q_{\mu} g_{h}^{(\mu)} \cap A_{\mu} \leqq g_{j}^{(\mu)} Q_{\mu} g_{h}^{(\mu)} \cap R_{\mu}
$$


for all $j, h=1,2, \ldots, n_{\mu}$. Therefore there are $n_{\mu}-1$ elements $q_{i}^{(\mu)} \in Q_{\mu}\left(i=1,2, \ldots, n_{\mu}-1\right)$ such that for all $i$ we have

$$
0 \neq g_{i}^{(\mu)} q_{i}^{(\mu)} g_{i+1}^{(\mu)} \in g_{i}^{(\mu)} Q_{\mu} g_{i+1}^{(\mu)} \cap A_{\mu} \leqq T_{\mu} \leqq G_{\mu} .
$$

Since $G_{\mu}=\left(G+P_{\mu}\right) / P_{\mu}, G_{\mu}$ is a nil subring of the prime right Goldie ring $R_{\mu}$, because each $\mathrm{n}$-ring is a nil ring. Hence $G_{\mu}$ is nilpotent. Clearly

$$
t_{\mu}=\sum_{i=1}^{n_{\mu}-1} g_{i}^{(\mu)} q_{i}^{(\mu)} g_{i+1}^{(\mu)} \in T_{\mu} \leqq G_{\mu} \leqq R_{\mu} .
$$

Hence, by application of Lemma $3(b)$ (ii) to the prime right Goldie ring $R_{\mu}, G_{\mu} \leqq F_{\mu}$. By the right-left symmetry of this argument we get $H_{\mu} \leqq E_{\mu}$.

Now

$$
G \leqq \sum_{\mu \in I}^{c} G_{\mu} \leqq \sum_{\mu \in I}^{c} F_{\mu}, \quad \text { and } \quad H \leqq \sum_{\mu \in I}^{c} H_{\mu} \leqq \sum_{\mu \in I}^{c} E_{\mu} .
$$

Therefore $G \cap H=0$, because $G_{\mu} \cap F_{\mu}=0$ for all $\mu \in I$. This completes the proof of Lemma 4 .

Before stating our Theorem 1 we restate some definitions of [11]. Let $\mathfrak{L}$ be the universal class of all [associative] rings; then a single-valued function $f$ assigning to every ring $R$ a (two-sided) ideal $f R$ of $R$ is called a preradical over $\mathfrak{U}$ if it satisfies

$$
(f R)^{\mu} \leqq f R^{\mu} \text { for every epimorphism } \mu \text { of } R \text {. }
$$

It is well known (cf. Jacobson [6]) that the lower and the upper nil radical, the Levitzkiradical and the Jacobson-radical are preradicals.

Let $f$ and $g$ be preradicals over $\mathfrak{l l}$. Then we define $f \leqq g$ if and only if $f R \leqq g R$ for all rings $R$. If $f$ is a preradical over $\mathfrak{U}$, we term a ring $S$ an $f$-ring if $S=f S$. The ideal $X$ of the ring $R$ is an $f$-ideal of $R$ if $X$ is an $f$-ring. We denote by $s_{f} R$ the sum of all $f$-ideals of the ring $R$. Using these definitions and notations we now establish the following theorem.

THEOREM 1. Let $f$ be a preradical over $\mathfrak{l l}$ with the following properties:

A. If $L$ is the Levitzki-radical and $N$ the upper nil radical over $\mathfrak{U}$, then $L \leqq f \leqq N$.

B. Extensions of $f$-rings by f-rings are f-rings.

C. The union of each tower of $f$-rings is an f-ring.

If $R / s_{f} R$ is a generalized right Goldie ring, then the sum $s_{f} R$ of all $f$-ideals of the ring $R$ is the intersection of all maximal $f$-subrings of $R$.

Proof. Let $t$ be a tower of $f$-ideals $X$ of $R$. If $T=\sum_{X \in \mathfrak{t}} X$, then $T$ is an $f$-ideal by C. Hence there exists a maximal $f$-ideal $M$ of $R$ by Zorn's Lemma. If $M \neq s_{f} R$, then there would be an $f$-ideal $Y$ of $R$ with $Y \$ M$. Since $M$ is an $f$-ring, we would have

$$
f[M / M \cap Y]=M / M \cap Y \cong(M+Y) / Y .
$$


Hence $M+Y$ would be an $f$-ideal of $R$, by B. Thus $M$ would not be a maximal $f$-ideal of $R$. This contradiction proves that $M=s_{f} R$. Since $s_{f} R$ is an $f$-ideal of $R$, the only $f$-ideal of $R / s_{f} R$ is 0 , by C. From $L \leqq f$ we obtain that $R / s_{f} R$ is a semi-prime ring.

Using $\mathrm{C}$, another application of Zorn's Lemma establishes the existence of maximal $f$-subrings of $R$. Now, if $F$ is such a subring of $R, s_{f} R \leqq F$. Hence from B it follows that the subring $S$ of $R$ is a maximal $f$-subring of $R$ if and only if $S \geqq s_{f} R$ and $S / s_{f} R$ is a maximal $f$-subring of $\bar{R}=R / s_{f} R$. Thus, if $F_{\alpha}(\alpha \in \bar{A})$ are the maximal $f$-subrings of $R$, then the $F_{\alpha} / s_{f} R$ are the maximal $f$-subrings of $\bar{R}$. Since $\bar{R}$ is a semi-prime generalized right Goldie ring, the intersection of all maximal $f$-subrings of $\bar{R}$ is zero by Lemma 4 . Hence

which implies that

$$
\left(\bigcap_{\alpha \in \bar{A}} F\right) / s_{f} R=\bigcap_{\alpha \in \bar{A}}\left[F_{\alpha} / s_{f} R\right]=0,
$$

$$
\bigcap_{\alpha \in \bar{A}} F_{\alpha}=s_{f} R
$$

This completes the proof of Theorem 1.

By Lemma 1 and Jacobson [6, p. 197, Lemma and Proposition 1, and p. 193, Lemma 1], the Levitzki-radical $L$ and the upper nil radical $N$ over $\mathfrak{U}$ satisfy the conditions $\mathrm{A}, \mathrm{B}$ and $\mathrm{C}$ of Theorem 1. Hence we have

COROLlaRY 2. (a) If $R / L(R)$ is a generalized right Goldie ring, then the sum $L(R)$ of all locally nilpotent ideals of $R$ is the intersection of all maximal locally nilpotent subrings of $R$.

(b) If $R / N(R)$ is a generalized right Goldie ring, then the sum $N(R)$ of all nil ideals of $R$ is the intersection of all maximal nil subrings of $R$.

COROLlaRY 3. Let $B(R)$ be the lower nil radical of the ring $R$. Let $R / B(R)$ be a generalized right Goldie ring with the following property:

$\left(b^{\prime}\right)$ Each set of independent, uniform right ideals of $R$ which are subisomorphic in pairs has at most $n$ elements, where $n$ is a fixed positive integer.

Then the following properties of the ring $R$ are equivalent:

(i) $B(R)$ is nilpotent.

(ii) $B(R)$ is the intersection of all maximal nilpotent subrings of $R$.

Proof. Clearly (i) is a consequence of (ii). Assume that $R$ satisfies condition (i). Since $R / B(R)$ is a semi-prime generalized right Goldie ring the Levitzki-radical $L(R)$ coincides with $B(R)$, by $[10$, Zusatz 5.4]. Hence $B(R)$ is the intersection of all maximal locally nilpotent subrings of $R$, by Corollary $2(a)$. Let $M$ be a maximal locally nilpotent subring of $R$. Then $M / B(R)=\bar{M}$ is a maximal locally nilpotent subring of $R / B(R)=\bar{R}$. By [10, Theorem 4.8] $\bar{R}$ is a subring of a complete direct sum $\bar{Q}$ of complete rings of $n_{\mu} \times n_{\mu}$ matrices over division rings $K_{\mu}(\mu \in I)$. From $\left(b^{\prime}\right)$ one easily deduces that $n_{\mu} \leqq n$ for all $\mu \in I$. Hence $\bar{M}$ is nilpotent by application of Lemma 2(a). This completes the proof of Corollary 3. 
COROLlaRY 4. The lower nil radical $B(R)$ of a right Noetherian ring is the intersection of all maximal nilpotent subrings of $R$.

This follows at once from Corollary 3.

THEOREM 2. If $R$ is a right Artinian ring without additive subgroups of type $p^{\infty}$, then the maximal nilpotent subrings of $R$ are quasi-conjugate.

Proof. Since $R^{+}$does not contain any subgroup of type $p^{\infty}$, it follows from Fuchs $[3$, p. 283, Theorem 73.1$]$ that

$$
R=C_{0} \oplus C_{1} \oplus C_{2} \oplus \ldots \oplus C_{r},
$$

where $C_{0}$ is a torsion-free right Artinian ring, and the $C_{i}(i=1,2, \ldots, r)$ are uniquely determined $\left(p_{i} \neq p_{j}\right.$, if $\left.i \neq j\right)$ right Artinian $p_{i}$-rings whose elements are of bounded order.

Let $U_{0}=\left\{(c, \mu) \mid c \in C_{0}, \mu \in Q\right.$, where $Q$ is the field of rationals $\}$, where the addition is defined componentwise and the multiplication by

$$
\left(c_{1}, \mu_{1}\right)\left(c_{2}, \mu_{2}\right)=\left(c_{1} c_{2}+\mu_{1} c_{2}+\mu_{2} c_{1}, \mu_{1} \mu_{2}\right)
$$

Then, by Fuchs $[3, \mathrm{p} .284], U_{0}$ is a unitary right Artinian ring such that $C_{0}$ is an ideal of $U_{0}$.

For $i=1,2, \ldots, r$ let $p_{i}^{k_{t}}$ be the least upper bound of the orders of the elements of $C_{i}$. Let $Z$ be the ring of rational integers, and let $Z\left(p_{i}^{k_{i}}\right)$ be the factor $\operatorname{ring}$ of $Z \bmod p_{i}^{k_{i}}$. Let

$$
U_{i}=\left\{(c, \mu) \mid c \in C_{i}, \mu \in \mathbb{Z}\left(p_{i}^{k_{l}}\right)\right\},
$$

where addition is defined componentwise and the multiplication by (3.2). Then, by Fuchs $\left[3\right.$, p. 285], each $U_{i}(i=1,2, \ldots, r)$ is a unitary right Artinian ring such that $C_{i}$ is a twosided ideal of $U_{i}$. Hence

$$
U=U_{0} \oplus U_{1} \oplus U_{2} \oplus \ldots \oplus U_{r}
$$

is a right Artinian ring. Since each right ideal of $R$ is a right ideal of $U, R$ is right Noetherian. Thus $R$ has maximal nilpotent subrings by Proposition 1. If $N$ is such a subring of $R$, then the $C_{j}$-component $(j=0,1,2, \ldots, r)$

$$
N_{j}=\left\{x \in R \mid x=n-y \in C_{j} \text { for some } n \in N \text { and } y \in \sum_{k \neq j} U_{k}\right\}
$$

of $N$ is a maximal nilpotent subring of $C_{j}$, and

$$
N=N_{0} \oplus N_{1} \oplus \ldots \oplus N_{r}
$$

by Barnes [1, p. 234, Lemma 1], because $R$ is right Artinian. Let $M$ be another maximal nilpotent subring of $R$. Then

$$
M=M_{0} \oplus M_{1} \oplus \ldots \oplus M_{r}
$$

G 
Since $U_{0} / C \cong Q$, all nilpotent subrings of $U_{0}$ are contained in $C_{0}$. Hence $N_{0}$ and $M_{0}$ are maximal nilpotent subrings of the unitary right Artinian ring $U_{0}$. Thus, by Köthe $[7, \mathrm{p} .363$, Theorem 5], there is a unit $u_{0} \in U_{0}$ satisfying

$$
M_{0}=u_{0}^{-1} N_{0} u_{0} .
$$

Let $u_{0}=\left(c_{0}, \mu_{0}\right)$ and $u_{0}^{-1}=\left(d_{0}, \mu_{0}^{-1}\right)$.

If $q_{0}=\left(-c_{0}, 0\right)\left(0, \mu_{0}^{-1}\right)$ and $p_{0}=\left(-d_{0}, 0\right)\left(0, \mu_{0}\right)$, then $p_{0}$ is the quasi-inverse of $q_{0}$ in $C_{0}$, and we have

$$
M_{0}=\left(1-p_{0}\right) N_{0}\left(1-q_{0}\right) .
$$

For $j=1,2, \ldots, r$ we know that $N_{j}$ (resp. $M_{j}$ ) is a maximal nilpotent subring of $C_{j}$. Let $J_{j}$ be the radical of $Z\left(p_{j}^{k_{j}}\right)$. Then

$$
N_{j}^{*}=\left(N_{j}, 0\right)+\left(0, J_{j}\right)
$$

is a nilpotent subring of $U_{j}$. If $N_{j}^{*}$ were not a maximal nilpotent subring of $U_{j}$, then there would be an element $v \in U_{j}$ such that $\left\{N_{j}^{*}, v\right\}$ is nilpotent.

Hence $v=\left(c_{j}, z_{j}\right)\left[c_{j} \in C_{j}, z_{j} \in Z\left(p_{j}^{k_{j}}\right)\right]$ is nilpotent. This implies that $z_{j} \in J_{j}$, and

$$
c_{j}=\left(c_{j}, 0\right)=v-\left(0, z_{j}\right) \in\left\{N_{j}^{*}, v\right\} \cap C_{j} \text {. }
$$

Now $\left\{N_{j}, c_{j}\right\} \leqq\left\{N_{j}^{*}, v\right\}$ and the fact that $N_{j}$ is a maximal nilpotent subring of $C_{j}$ imply that $c_{j} \in N_{j}$, which implies that $v \in N_{j}^{*}$, a contradiction. Hence $N_{j}^{*}$ is a maximal nilpotent subring of $U_{j}$. By the same argument we obtain that $M_{j}^{*}=\left(M_{j}, 0\right)+\left(0, J_{j}\right)$ is a maximal nilpotent subring of $U_{j}$. Thus, by Köthe [7, p. 363, Theorem 5], there is a unit $u_{j} \in U_{j}$ satisfying

$$
M_{j}^{*}=u_{j}^{-1} N_{j}^{*} u_{j} .
$$

Now identify $M_{j}$ with $\left(M_{j}, 0\right)$ and $N_{j}$ with $\left(N_{j}, 0\right)$. Then it is obvious that $u_{j}^{-1} N_{j} u_{j} \leqq M_{j}$. Conversely, for each $m_{j}$ there is an $n_{j}^{*}=n_{j}+z_{j}$, where $n_{j} \in N_{j}, z_{j} \in J_{j}$, such that

$$
m_{j}=u_{j}^{-1}\left(n_{j}+z_{j}\right) u_{j}=u_{j}^{-1} n_{j} u_{j}+u_{j}^{-1} z_{j} u_{j} .
$$

Let $u_{j}=d_{j}+r_{j}, u_{j}^{-1}=e_{j}+s_{j}$, where $d_{j}, e_{j} \in N_{j}, r_{j}, s_{j} \in Z\left(p_{j}^{k_{j}}\right)$ and $r_{j} s_{j}=1=s_{j} r_{j}$. Then it follows that

$$
m_{j}-u_{j}^{-1} n_{j} u_{j}-z_{j} e_{j} d_{j}-s_{j} z_{j} d_{j}-r_{j} z_{j} e_{j}=s_{j} z_{j} r_{j} \in C_{j} \cap Z\left(p_{j}^{k j}\right)=0 .
$$

Hence $z_{j}=0$. This means that $M_{j}=u_{j}^{-1} N_{j} u_{j}$.

For each $j=1,2, \ldots, r$, let $q_{j}=\left(-d_{j}, 0\right)\left(0, r_{j}^{-1}\right)$ and $p_{j}=\left(-e_{j}, 0\right)\left(0, r_{j}\right)$. Then $p_{j}$ is the quasi-inverse of $q_{j}$ in $C_{j}$, and we have $M_{j}=\left(1-p_{j}\right) N_{j}\left(1-q_{j}\right)$. If $p=\sum_{k=0}^{r} p_{k}$ and $q=\sum_{k=0}^{r} q_{k}$, then $p$ is the quasi-inverse of $q$ in $R$, and it follows that $M=(1-p) N(1-q)$. This completes the proof of Theorem 2 . 


\section{REFERENCES}

1. D. W. Barnes, On the radical of a ring with minimum condition, J. Australian Math. Soc. 5 (1965), 234-236. 53-60.

2. C. Faith and Y. Utumi, On Noetherian prime rings, Trans. Amer. Math. Soc. 114, (1965),

3. L. Fuchs, Abelian groups (Oxford, London, New York, Paris, 1960).

4. A. W. Goldie, Semi-prime rings with maximum condition, Proc. London Math. Soc. Ser. 310 (1960), 201-220.

5. I. N. Herstein and L. Small, Nil rings satisfying certain chain conditions, Canad. J. Math. 16 (1964), 771-776. 1964).

6. N. Jacobson, Structure of rings, Amer Math. Soc. Colloquium Publications 37 (Providence, 359-363.

7. G. Köthe, Über maximale nilpotente Unterringe und Nilringe, Math. Ann. 103 (1930),

8. L. Levitzki, Über nilpotente Unterringe, Math. Ann. 105 (1931), 620-627.

9. L. Levy, Unique subdirect sums of prime rings, Trans. Amer. Math. Soc. 106 (1963), 64-76.

10. G. Michler, Klassische Quotientenringe von nicht notwendig endlich dimensionalen, halbprimen Ringen, Math. $Z$. (to appear).

11. G. Michler, Radikale und Sockel, accepted for publication by Math. Ann.

12. C. Procesi and L. Small, On a theorem of Goldie, J. Algebra 2 (1965), 80-84.

Added in proof. We now have an example of a prime right and left Goldie ring with identity having two maximal milpotent subrings which are not isomorphic (cf Michler, Math. $Z .100$ (1967), p. 180).

JOHANN WOLFGANG GOETHE UNIVERSITÄT

FRANKFURT 